\title{
Wave-particle resonance condition test for ion-kinetic waves in the solar wind
}

\author{
Y. Narita ${ }^{1,2}$, E. Marsch ${ }^{3}$, C. Perschke ${ }^{2,4}$, K.-H. Glassmeier ${ }^{2,5}$, U. Motschmann ${ }^{4,6}$, and H. Comişel ${ }^{4,7}$ \\ ${ }^{1}$ Space Research Institute, Austrian Academy of Sciences, Schmiedlstr. 6, 8042 Graz, Austria \\ ${ }^{2}$ Institut für Geophysik und extraterrestrische Physik, Technische Universität Braunschweig, \\ Mendelssohnstr. 3, 38106 Braunschweig, Germany \\ ${ }^{3}$ Institut für Experimentelle und Angewandte Physik, Christian-Albrechts Universität Kiel, \\ Leibnizstr. 11, 24118 Kiel, Germany \\ ${ }^{4}$ Institut für Theoretische Physik, Technische Universität Braunschweig, Mendelssohnstr. 3, 38106 Braunschweig, Germany \\ ${ }^{5}$ Max-Planck-Institut für Sonnensystemforschung, Justus-von-Liebig-Weg 3, 37077 Göttingen, Germany \\ ${ }^{6}$ Deutsches Zentrum für Luft- und Raumfahrt, Institut für Planetenforschung, Rutherfordstr. 2, 12489 Berlin, Germany \\ ${ }^{7}$ Institute for Space Sciences, Atomiştilor 409, P.O. Box MG-23, Bucharest-Măgurele, 077125, Romania
}

Correspondence to: Y. Narita (yasuhito.narita@oeaw.ac.at)

Received: 8 December 2015 - Revised: 19 March 2016 - Accepted: 4 April 2016 - Published: 7 April 2016

\begin{abstract}
Conditions for the Landau and cyclotron resonances are tested for 543 waves (identified as local peaks in the energy spectra) in the magnetic field fluctuations of the solar wind measured by the Cluster spacecraft on a tetrahedral scale of $100 \mathrm{~km}$. The resonance parameters are evaluated using the frequencies in the plasma rest frame, the parallel components of the wavevectors, the ion cyclotron frequency, and the ion thermal speed. The observed waves show a character of the sideband waves associated with the ion Bernstein mode, and are in a weak agreement with the fundamental electron cyclotron resonance in spite of the ionkinetic scales. The electron cyclotron resonance is likely taking place in solar wind turbulence near $1 \mathrm{AU}$ (astronomical unit).
\end{abstract}

Keywords. Space plasma physics (wave-particle interactions)

\section{Introduction}

Wave-particle interactions are of particular importance in collisionless plasmas in space and astrophysical systems. Various types of interactions are possible, e.g., Landau resonance, cyclotron resonance and its harmonic resonances, and pitch-angle scattering. Wave-particle interaction is the candidate process to explain dissipation in plasma turbulence and anomalous resistivity in ionospheric heating processes (Coroniti, 1985), plays potentially an important role in driving magnetic reconnection (Treumann, 2001), and is of importance for wave excitation through microinstabilities due to the unstable gradient of the velocity distribution function such as beams and temperature anisotropies as studied in detail in theory by Gary (1993) and in spacecraft observations by Marsch (2006).

The Landau and cyclotron resonances are an essential ingredient in the wave and instability analysis in the linear Vlasov theory. Waves and particles are in resonance when the apparent field-aligned phase speed of the wave (corrected for the cyclotron frequency or its harmonics) is sufficiently close to the thermal speed of the particles, namely when the condition

$\zeta^{(m)}=\frac{\omega-m \Omega_{s}}{k_{\|} v_{\mathrm{th}}} \sim 1$

holds. Here $\omega$ denotes the frequency, $\Omega_{s}$ the cyclotron frequency of species $s, m$ the harmonic degree of resonance $(m=0, \pm 1, \pm 2, \ldots), k_{\|}$the parallel (or field-aligned) component of the wavevector in the direction to the mean magnetic field, and $v_{\text {th }}$ the thermal speed of species $s$. It should be noted here that the apparent phase speed $\omega / k_{\|}$is involved in the resonance condition, not the total phase speed $\omega /|\boldsymbol{k}|$ and not the field-aligned component of the phase speed $\omega k_{\|} /|\boldsymbol{k}|^{2}$. Equation (1) serves as a measure of the Landau resonance for 
$m=0$, the fundamental cyclotron resonance for $m=1$, and the cyclotron harmonic resonance for the other values of $m$. As is seen in Eq. (1), the measurement of the resonance parameter $\zeta^{(m)}$ requires knowledge of the frequencies in the plasma rest frame and the wavevectors, which is not feasible using single-spacecraft measurements only.

Here we present an observational study of the Landau and cyclotron resonance conditions using the database for the low-frequency wave samples of magnetic field fluctuations in the solar wind on the spatial scales of ion kinetics (at about the inertial length and smaller) (Perschke et al., 2014). Our study presents for the first time that the Landau and the cyclotron resonance conditions are evaluated directly and observationally for protons and electrons. The difficulty in obtaining knowledge on the rest-frame frequencies and the wavevectors is overcome by using four-spacecraft measurements performed on board Cluster (Escoubet et al., 2001) in the solar wind near $1 \mathrm{AU}$.

\section{Wave-particle resonance test}

\subsection{Kinetic-wave database}

The database originates from four-point magnetic field measurements performed by the fluxgate magnetometers on board Cluster (Balogh et al., 2001). The database represents a collection of the wave samples at the smallest tetrahedral spatial scales of about $100 \mathrm{~km}$ in the solar wind with the full three-dimensional spatial resolution (from February to April 2002). That scale is ideal for studying waves on the ionkinetic scale because the ion inertial length is of the order of a few hundred kilometers. Nine time intervals are identified as suited for the wave analysis, of which 543 discrete wave samples (local peaks in the energy spectra) are registered into the wave database. The values of ion beta are in the range $0.5 \leq \beta_{\mathrm{i}} \leq 1.6$ with a mean value of about 1.01 . Wave propagation angles are quasi-perpendicular from the mean magnetic field.

In the database, nine intervals of the pristine solar wind measurements are used under a condition of quasi-stationary and homogeneous mean magnetic and flow velocity fields, e.g., change of the mean magnetic field up to $15^{\circ}$, fluctuation amplitude relative to the mean magnetic field being at most a few percent, and that to the mean flow velocity smaller than $1 \%$ (Perschke et al., 2014). A typical example of the frequency spectrum is shown in Fig. 1.

The method of the wave analysis is the Multi-point Signal Resonator (MSR) algorithm (Narita et al., 2011), which makes use of the minimum variance projection (maximum likelihood estimate for a Gaussian likelihood function) and the eigenvector-based adaptive projection called the extended MUSIC (Multiple Signal Classification) algorithm. In the MSR algorithm, isotropic fluctuations are regarded as noise, and coherent wavevectors are selected for the further analy-

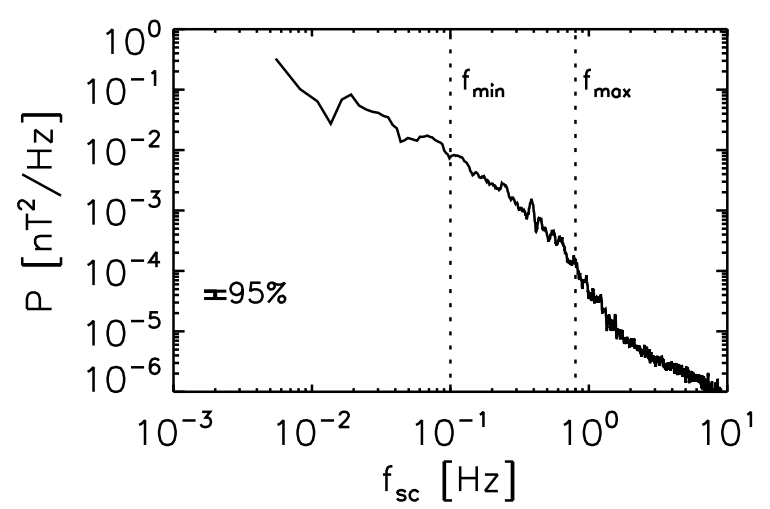

Figure 1. Frequency spectrum for magnetic field fluctuation in the solar wind measured by Cluster-1 on 11 February 2002, 15:2915:38 UT, from the kinetic-wave database in Perschke et al. (2014). The lower and the upper border frequencies for the wave analysis are indicated by as $f_{\min }$ and $f_{\max }$.

sis. We pick up the wave components that are persistent or coherent in the data with a larger fluctuation amplitude without assuming a particular dispersion relation in the data. The MSR algorithm detects waves that have amplitudes larger than the isotropic fluctuation level (we refer to it as a noise level). One may expect that strong turbulence exhibits a broadband spectrum, and in our analysis, small-amplitude fluctuations below the isotropic level are not considered for the study.

From a first application or analysis of the database, Perschke et al. (2014) have concluded that the wave frequencies in the plasma rest frame are broadly distributed from nearly zero to over the ion cyclotron frequency and appear as sideband waves around the theoretical frequencies of the linear modes. Before applying the resonance condition test to the database, we first revisit the wave frequencies (or the dispersion relation diagram) in view of the kinetic slow mode which has recently been found to be competitive as a possible wave mode in solar wind turbulence (Howes et al., 2012; Klein et al., 2012; Narita and Marsch, 2015). In the analysis, the wave frequencies are transformed from the spacecraft frame into the plasma rest frame by correcting for the Doppler shift, $\boldsymbol{k} \cdot \boldsymbol{U}_{\mathrm{sw}}$, where $\boldsymbol{k}$ and $\boldsymbol{U}_{\mathrm{sw}}$ are the wavevector obtained from the multi-point wave analysis (Narita et al., 2011) and the ion bulk velocity obtained from the Cluster electrostatic ion analyzer instruments (Rème et al., 2001), respectively.

The top panel in Fig. 2 displays the dispersion relation diagram, a plot of wave frequencies (in the plasma rest frame) as a function of the wavevector magnitudes. The wave samples are separated into the high-beta group $\left(\beta_{\mathrm{i}} \geq 1\right)$ and the lowbeta group $\left(\beta_{\mathrm{i}}<1\right)$. The frequencies and the wavevectors are normalized to the ion cyclotron frequency $\Omega_{\mathrm{p}}$ (for protons) and the ion inertial length $V_{\mathrm{A}} / \Omega_{\mathrm{p}}$, respectively (here $V_{\mathrm{A}}$ is the Alfvén speed). The normal modes in the linear Vlasov 


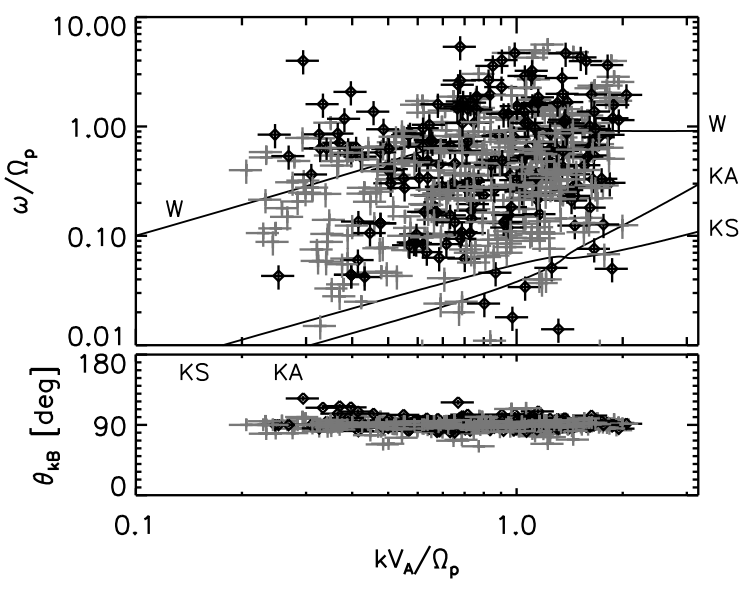

Figure 2. Top panel: scatter plot of wave frequencies in the plasma rest frame as a function of the wavevector magnitude for the discrete waves (identified as the local peaks in the energy spectra) observed by the Cluster spacecraft (Perschke et al., 2014). Data points in gray diamond indicate low-beta conditions $\left(\beta_{\mathrm{i}}<1\right)$ that in black cross high-beta conditions $\left(\beta_{\mathrm{i}} \geq 1\right)$ in the solar wind. Theoretical dispersion relations for whistler mode $(\mathrm{W})$, kinetic slow mode $(\mathrm{KS})$, and kinetic Alfvén mode (KA) for $\beta_{\mathrm{i}}=1$ and $\theta_{\mathrm{kB}}=88^{\circ}$ are overplotted. Bottom panel: wavevector angles from the mean magnetic field.

theory are calculated using the dispersion solver described in Gary (1993) (or in Narita and Marsch, 2015 for the detailed implementation), and are over-plotted under the typical condition of the measured solar wind: ion beta of unity $\beta_{\mathrm{i}}=1$ and the quasi-perpendicular wavevectors at an angle of $88^{\circ}$ from the mean magnetic field (set by the measured wavevector angles, see the bottom panel and the next paragraph).

Most of the wave frequencies show a deviation from that of the linear modes. The frequencies are too high to be explained as the kinetic Alfvén mode or the kinetic slow mode. However, the frequencies are more concentrated around the ion Bernstein mode (as the high-wavenumber branch of the whistler mode at about the ion cyclotron frequency, $\omega / \Omega_{\mathrm{p}} \simeq$ 1). There is no clear indication that wave modes are better identified for higher or lower values of ion beta. Therefore, we obtain the results that there is a weak indication of the ion Bernstein mode and that the kinetic slow mode is not likely the best candidate to explain the measured solar wind fluctuations irrespective of ion beta (in the measured range of beta, of course).

The bottom panel in Fig. 2 shows a scatter plot of the wavevector angles from the mean magnetic field $\theta_{\mathrm{kB}}$. The wavevectors are quasi-perpendicular to the mean field. The mean angle with the standard deviation as an error bar range is $90.2^{\circ} \pm 6.2^{\circ}$ when computed in the range up to $180^{\circ}$, and $86.1^{\circ} \pm 4.8^{\circ}$ in the range up to $90^{\circ}$ (by measuring the angles either from the mean field or from the anti-parallel direction to the mean field), which motivates setting the angle $\theta_{\mathrm{kB}}=88^{\circ}$ for the dispersion relation test in the top panel.

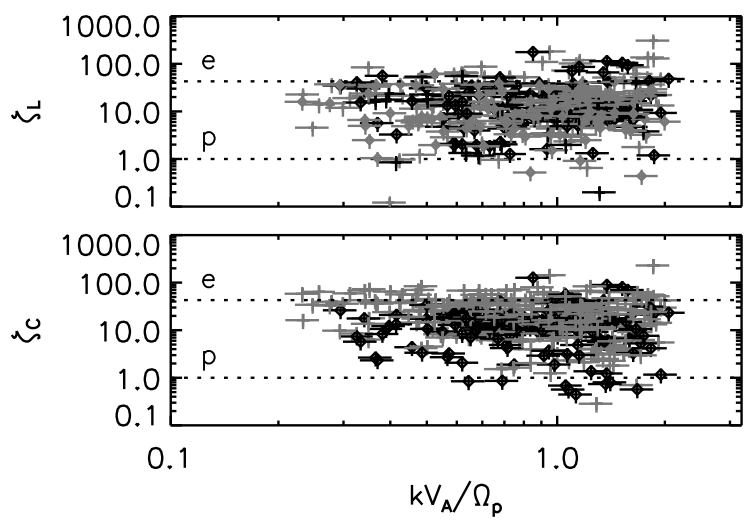

Figure 3. Landau resonance parameter with protons $\zeta_{\mathrm{L}}$ and cyclotron resonance parameter with protons $\zeta_{\mathrm{C}}$ for the data set presented in Fig. 2. The resonance line for protons are marked by "p", and that for electrons are marked by "e" multiplied by a factor of $\sqrt{m_{\mathrm{p}} / m_{\mathrm{e}}} \simeq 42.85$.

Errors are estimated at each data point in Fig. 2 as follows: relative error of the wavenumbers is $10 \%$ (grid size in the wavenumber domain); that of the mean flow velocities is up to $15 \%$ (flow statistics); that of the rest-frame frequency is up to $25 \%$; (on Doppler shift estimate) (Perschke et al., 2014); that of the resonance parameter is up to $35 \%$; and that of the wavevector angle is $3^{\circ}$.

\subsection{Landau and ion cyclotron resonance test}

The resonance conditions are tested for the Landau and cyclotron types for protons and electrons using the database. The proton Landau resonance condition is evaluated for $m=$ 0 in Eq. (1), and the ion cyclotron resonance condition for $m= \pm 1$ in Eq. (1), denoted by $\zeta_{\mathrm{L}}$ and $\zeta_{\mathrm{C}}$, respectively. Here, both signs are used in the ion-cyclotron resonance study because the rest-frame frequencies can be both positive (propagating in the direction away from the Sun in the rest frame) and negative (toward the Sun). The thermal speed of protons is obtained by the Cluster electrostatic ion analyzer instruments (Rème et al., 2001). The resonance conditions for thermal electrons (assuming the same temperature between ions and electrons) are obtained by multiplying the proton resonance conditions by a factor of $\sqrt{m_{\mathrm{e}} / m_{\mathrm{p}}} \simeq(42.85)^{-1}$. Equivalently, one may plot a compensated resonance parameter by multiplying the proton resonance parameter by the factor 42.85 in the diagram of the proton resonance parameters to study if the electron resonance condition is satisfied.

The Landau and the cyclotron resonance parameters for protons are plotted as a function of the wavenumbers in Fig. 3. Both the Landau and cyclotron resonance parameters show a distribution in a broad range of the wavenumbers. The distribution of the Landau resonance parameter is concentrated well above the resonance with the protons $(\zeta \mathrm{L}(\mathrm{p}) \gg 1)$ yet slightly below that with the electrons. The 

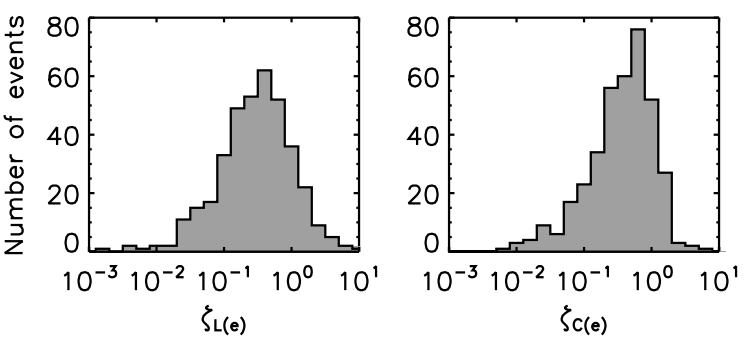

Figure 4. Histogram of electron Landau resonance parameter $\zeta_{\mathrm{L}(\mathrm{e})}$ (left panel) and cyclotron resonance parameter $\zeta_{\mathrm{C}(\mathrm{e})}$.

distribution of the cyclotron resonance parameter is also concentrated well above the resonance with the protons and nearly satisfied the resonance condition for the electrons $\zeta_{\mathrm{C}(\mathrm{e})} \simeq \zeta_{\mathrm{C}(\mathrm{p})} \sqrt{m_{\mathrm{e}} / m_{\mathrm{p}}} \sim 1$. There is only weak dependence of the measured resonance parameters on the wavenumbers. Also, there is only weak dependence on the values of plasma beta.

Figure 4 shows the distributions of the electron resonance parameters in a histogram style. A peak is found between 10 and $100 \%$ of the resonance condition for the both electron Landau and cyclotron resonances. Furthermore, the distribution of the electron cyclotron resonance peaks nearly at $\zeta_{\mathrm{C}(\mathrm{e})} \simeq 1$. To summarize, coherent waves in the solar wind do not satisfy the resonance condition for the protons, but satisfy reasonably the condition for the electrons. In particular, the electron cyclotron resonance is likely in operation.

There is no clear separation between the low-beta group and the high-beta group in the resonance parameter study except for the weak separation in the Landau resonance parameter that the high-beta group (in black) appears to have slightly smaller values (in the range $1<\zeta_{\mathrm{L}}<10$ ) than that for the low-beta group (in gray, in the range $10<\zeta_{\mathrm{L}}<100$ ).

\section{Discussion and outlook}

We find the wave modes to appear mostly as sideband waves with a weak indication to whistler and ion Bernstein modes. A detailed wave mode study by Perschke et al. (2014) using the kinetic-data base shows that both linear modes such as whistler, Bernstein, and kinetic modes and sideband waves exist in the solar wind. On the other hand, there have been recent studies suggesting that the kinetic Alfvén mode is dominant in the solar wind, e.g., from the k-filtering technique (Sahraoui et al., 2010; Roberts et al., 2013) and by other techniques (Salem et al., 2012; TenBarge et al., 2012; Kiyani et al., 2013; Chen et al., 2013). A question arises naturally as the reason why the kinetic Alfvén mode does not clearly appear in our kinetic-wave database. A possible explanation includes a scenario that sideband waves play a more fundamental role in solar wind turbulence. The MSR algorithm is more sensitive than existing multi-spacecraft interferometric meth- ods such as k-filtering or wave telescope and can identify the wavevectors for coherent wave components more clearly. That is, the existence of multiple sideband waves may mislead the previous wave analyses to a different wave mode. A further test would be needed to verify this scenario.

The lesson from the wave-particle resonance test using the Cluster data in the sola wind is that non-normal or sideband waves play an important role in that even fluctuations on the ion-kinetic scales can potentially heat the electrons, which may be regarded as a realization of cross-scale coupling in a plasma. To achieve such a cross-scale coupling, the sideband waves must be continuously pumped by wavewave interactions within their lifetime, i.e., before the sideband wave changes its frequency and wavenumber through further wave-wave interactions.

Most of the major wave components in the solar wind do not show a clear organization of the dispersion relation. The wave frequencies appear as sideband and are distributed between that of the kinetic Alfvén or kinetic slow modes and that of whistler mode which undergoes transition into the Bernstein mode at higher wavenumbers. It is interesting that the sideband waves exhibit a weak indication of the electron cyclotron resonance in a broad range of wavenumbers around the ion inertial length $0.2<k V_{\mathrm{A}} / \Omega_{\mathrm{p}}<2$. In contrast, Landau or cyclotron resonance with ions is not a likely process near $1 \mathrm{AU}$ as wave frequencies or apparent field-aligned phase speeds are too high for the resonance with the thermal ions. We conclude, observationally speaking, that the electron heating through the cyclotron resonance with the quasiperpendicular propagating sideband waves is most relevant on ion-kinetic scales in the solar wind near $1 \mathrm{AU}$.

A clear demonstration for the existence of the electron cyclotron resonance requires further studies of the velocity distribution functions. Still, we may ask a question if the ion heating happens primarily in the inner heliosphere before the solar wind reaches $1 \mathrm{AU}$. The presence of ion pitchangle scattering is supported by the Helios spacecraft observations at 0.3 AU (Marsch and Bourouaine, 2011). On the other hand, there are other heating mechanisms apart from the resonances, for example stochastic heating or reconnection. Also, there might a possibility that wave modes with small amplitudes were not detected.

The question about the origin of the sideband waves remains unsolved. We speculate that the sideband waves are generated through wave-wave interactions (e.g., Verscharen et al., 2012; Comişel et al., 2013) or wave-particle-wave interactions (e.g., Marsch, 2006) associated with the normal modes. The frequencies deviate gradually from that of the normal modes such that the waves evolve into sideband waves in the dispersion relation diagram. Higher-order statistics for the fluctuating magnetic fields is ideal to test against three- or even four-wave resonance processes in the solar wind.

It is interesting to note that not so many particles in solar wind turbulence participate in the wave-particle interac- 
tions because the distributions of the resonance parameters are rather broad. The observational study of wave-particle interactions can be extended to the velocity space (the analysis of the velocity distribution functions) and to the smaller scales in the coordinate space down to the electron scales. Fortunately, the Magnetospheric MultiScale (MMS) mission (Burch et al., 2015) will encounter the solar wind when the spacecraft apogee is boosted to the solar wind for its second observational phase in the Earth magnetotail measurements. The combination study using both Cluster and MMS measurements will give us a more comprehensive picture about the dissipation mechanism in plasma turbulence.

Acknowledgements. The work by K.-H. Glassmeier is financially supported by the German Bundesministerium für Wirtschaft und Energie and the Deutsches Zentrum für Luft- und Raumfahrt under contract 50 OC 1402. The work by C. Perschke, U. Motschmann, and $\mathrm{H}$. Comişel is supported by Collaborative Research Center 963, "Astrophysical Flow, Instabilities, and Turbulence" of the German Science Foundation. The work conducted by $\mathrm{H}$. Comişel in Bucharest is supported by Romanian Ministry for Scientific Research and Innovation, CNCS - UEFISCDI, project no. PN-II-RUTE-2014-4-2420.

The topical editor, C. Owen, thanks one anonymous referee for help in evaluating this paper.

\section{References}

Balogh, A., Carr, C. M., Acuña, M. H., Dunlop, M. W., Beek, T. J., Brown, P., Fornacon, K.-H., Georgescu, E., Glassmeier, K.H., Harris, J., Musmann, G., Oddy, T., and Schwingenschuh, K.: The Cluster Magnetic Field Investigation: overview of in-flight performance and initial results, Ann. Geophys., 19, 1207-1217, doi:10.5194/angeo-19-1207-2001, 2001.

Burch, J. L., Moore, T. E., Torbert, R. B., and Giles, B. L.: Magnetospheric Multiscale overview and science objectives, Space Sci. Rev., doi:10.1007/s11214-015-0164-9, online first, 2015.

Chen, C. H. K., Boldyrev, S., Xia, Q., and Perez, J. C.: Nature of subproton scale turbulence in the solar wind, Phys. Rev. Lett., 110, 225002, doi:10.1103/PhysRevLett.110.225002, 2013.

Comişel, H., Verscharen, D., Narita, Y., and Motschmann, U.: Spectral evolution of two-dimensional kinetic plasma turbulence in the wavenumber-frequency domain, Phys. Plasmas, 20, 090701, doi:10.1063/1.4820936, 2013.

Coroniti, F. V.: Space plasma turbulent dissipation - Reality of myth?, Space Sci. Rev., 42, 399-410, 1985.

Escoubet, C. P., Fehringer, M., and Goldstein, M.: Introduction, The Cluster mission, Ann. Geophys., 19, 1197-1200, doi:10.5194/angeo-19-1197-2001, 2001.

Gary, S. P.: Theory of Space Plasma Microinstabilities, Cambrige Atmospheric and Space Science Series, Cambridge University Press, Cambridge, 1993.

Howes, G. G., Bale, S. D., Klein, K. G., Chen, C. H. K., Salem, C. S., and TenBarge, J. M.: The slow-mode nature of compressible wave power in solar wind turbulence, Astrophys. J. Lett., 753, L19, doi:10.1088/2041-8205/753/1/L19, 2012.
Kiyani, K. H., Chapman, S. C., Sahraoui, F., Hnat, B., Fauvarque, O., and Khotyaintsev, Y. V.: Enhanced magnetic compressibility and isotropic scale invariance at sub-ion Larmor scales in solar wind turbulence, Astrophys. J., 763, 10, doi:10.1088/0004637X/763/1/10, 2013.

Klein, K. G., Howes, G. G., TenBarge, J. M., Bale, S. D., Chen, C. H. K., and Salem, C. S.: Using synthetic spacecraft data to interpret compressible fluctuations in solar wind turbulence, Astrophys. J. 755, 159, doi:10.1088/0004-637X/755/2/159, 2012.

Marsch, E.: Kinetic physics of the solar corona and solar wind, Living Rev. Solar Phys., 3, 1, doi:10.12942/lrsp-2006-1, 2006.

Marsch, E. and Bourouaine, S.: Velocity-space diffusion of solar wind protons in oblique waves and weak turbulence, Ann. Geophys., 29, 2089-2099, doi:10.5194/angeo-29-2089-2011, 2011.

Narita, Y., Glassmeier, K.-H., and Motschmann, U.: Highresolution wave number spectrum using multi-point measurements in space - the Multi-point Signal Resonator (MSR) technique, Ann. Geophys., 29, 351-360, doi:10.5194/angeo-29-3512011, 2011.

Narita, Y. and Marsch, E.: Kinetic slow mode in the solar wind and its possible role in turbulence dissipation and ion heating, Astrophys. J., 805, 24, doi:10.1088/0004-637X/805/1/24, 2015.

Perschke, C., Narita, Y., Motschmann, U., and Glassmeier, K.H.: Multi-spacecraft observations of linear modes and sideband waves in ion-scale solar wind turbulence: Astrophys. J. Lett., 793, L25, doi:10.1088/2041-8205/793/2/L25, 2014.

Rème, H., Aoustin, C., Bosqued, J. M., Dandouras, I., Lavraud, B., Sauvaud, J. A., Barthe, A., Bouyssou, J., Camus, Th., Coeur-Joly, O., Cros, A., Cuvilo, J., Ducay, F., Garbarowitz, Y., Medale, J. L., Penou, E., Perrier, H., Romefort, D., Rouzaud, J., Vallat, C., Alcaydé, D., Jacquey, C., Mazelle, C., d'Uston, C., Möbius, E., Kistler, L. M., Crocker, K., Granoff, M., Mouikis, C., Popecki, M., Vosbury, M., Klecker, B., Hovestadt, D., Kucharek, H., Kuenneth, E., Paschmann, G., Scholer, M., Sckopke, N., Seidenschwang, E., Carlson, C. W., Curtis, D. W., Ingraham, C., Lin, R. P., McFadden, J. P., Parks, G. K., Phan, T., Formisano, V., Amata, E., Bavassano-Cattaneo, M. B., Baldetti, P., Bruno, R., Chionchio, G., Di Lellis, A., Marcucci, M. F., Pallocchia, G., Korth, A., Daly, P. W., Graeve, B., Rosenbauer, H., Vasyliunas, V., McCarthy, M., Wilber, M., Eliasson, L., Lundin, R., Olsen, S., Shelley, E. G., Fuselier, S., Ghielmetti, A. G., Lennartsson, W., Escoubet, C. P., Balsiger, H., Friedel, R., Cao, J.-B., Kovrazhkin, R. A., Papamastorakis, I., Pellat, R., Scudder, J., and Sonnerup, B.: First multispacecraft ion measurements in and near the Earth's magnetosphere with the identical Cluster ion spectrometry (CIS) experiment, Ann. Geophys., 19, 1303-1354, doi:10.5194/angeo19-1303-2001, 2001.

Roberts, O. W., Li, X., and Li, B.: Kinetic plasma turbulence in the fast solar wind measured by Cluster, Astrophys. J., 769, 58, doi:10.1088/0004-637X/769/1/58, 2013.

Sahraoui, F., Goldstein, M. L., Belmont, G., Canu, P., and Rezeau, L.: Three dimensional anisotropic $\mathrm{k}$ spectra of turbulence at subproton scales in the solar wind, Phys. Rev. Lett., 105, 131101, doi:10.1103/PhysRevLett.105.131101, 2010.

Salem, C. S., Howes, G. G., Sundkvist, D., Bale, S. D., Chaston, C. C., Chen, C. H. K., and Mozer, F. S.: Identification of kinetic Alfvèn wave turbulence in the solar wind, Astrophys. J. Lett., 745, L9, doi:10.1088/2041-8205/745/1/L9, 2012. 
TenBarge, J. M., Podesta, J. J., Klein, K. G., and Howes, G. G.: Interpreting magnetic variance anisotropy measurements in the solar wind, Astrophys. J., 753, 107, doi:10.1088/0004637X/753/2/107, 2012.

Treumann, R. A.: Origin of resistivity in reconnection, Earth Planets Space, 53, 453-462, doi:10.1186/BF03353256, 2001.
Verscharen, D., Marsch, E., Motschmann, U., and Müller, J.: Kinetic cascade beyond magnetohydrodynamics of solar wind turbulence in two-dimensional hybrid simulations, Phys. Plasmas, 19, 022305, doi:10.1063/1.3682960, 2012. 